\title{
Improved Biomimetic Total Synthesis of d,I-Stephacidin A
}

\author{
Thomas J. Greshock ${ }^{1}$ and Robert M. Williams ${ }^{1,2 *}$
}

${ }^{1}$ Department of Chemistry, Colorado State University, Fort Collins, Colorado 80523

${ }^{2}$ University of Colorado Cancer Center, Aurora, Colorado 80045

General Methods. Unless otherwise noted, all materials were obtained from commercial sources and used without purification. All reactions requiring anhydrous conditions were performed under a positive pressure of argon using flame-dried glassware. Dichloromethane, acetonitrile, toluene, and tetrahydrofuran were degassed with argon and dried through a solvent purification system (J.C. Meyer of Glass Contour). Flash chromatography was performed on standard grade silica gel ( $230 \times 400$ mesh) from Sorbent Technologies with the indicated solvent. ${ }^{1} \mathrm{H}$ NMR and ${ }^{13} \mathrm{C}$ NMR spectra were recorded on Varian 300 or $400 \mathrm{MHz}$ spectrometers as indicated. Infrared spectra were recorded on a Nicolet Avatar 320-FT IR spectrometer. Mass spectra were obtained at the Colorado State University CIF on a Fisons VG Autospec. Melting points were obtained on a Mel-Temp Laboratory Device. 

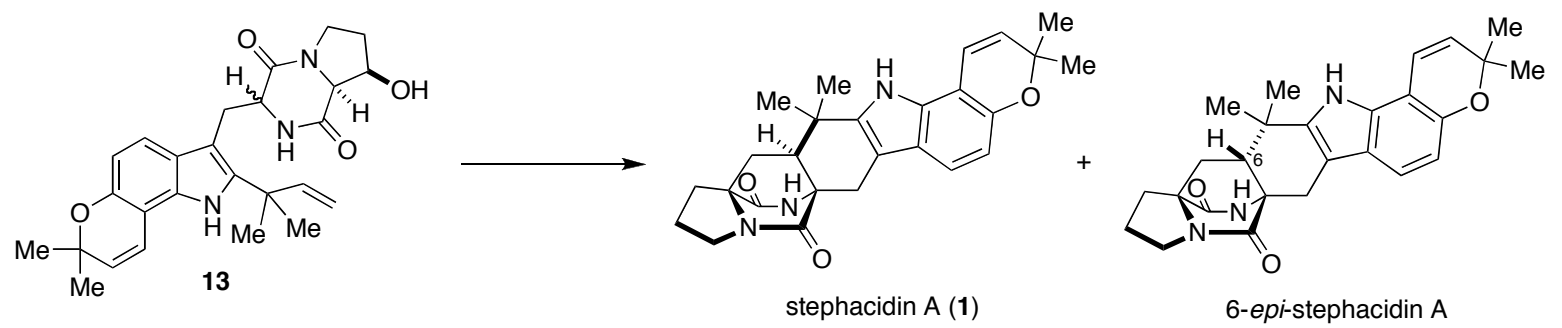

Stephacidin A (1). To a solution of alcohols $13(79 \mathrm{mg}, 0.18 \mathrm{mmol})$ in $\mathrm{CH}_{2} \mathrm{Cl}_{2}(18 \mathrm{~mL})$ at rt was added DEAD (166 $\mu \mathrm{L}, 1.06 \mathrm{mmol})$. The mixture was stirred at it for $5 \mathrm{~min}$ and $\mathrm{PBu}_{3}(264 \mu \mathrm{L}, 1.06 \mathrm{mmol})$ was then added. The solution was heated to $40^{\circ} \mathrm{C}$ for $20 \mathrm{~h}$. The resulting mixture was concentrated. Purification by silica gel chromatography (ethyl acetate : hexane, $1: 1$, then methanol : $\left.\mathrm{CH}_{2} \mathrm{Cl}_{2}, 5: 95\right)$ afforded stephacidin $\mathrm{A}(1)$ and 6 epi-stephacidin A as a $2.4: 1$ crude mixture of diastereomers, respectively. This mixture was triturated with methanol to afford stephacidin A (1) as a white solid (34.7 $\mathrm{mg}, 46 \%$ ). The methanol triturate was then concentrated to afford 6-epi-stephacidin A (13.5 mg, 18\%); All spectral data for stephacidin A (1) was identical to that previously reported; ${ }^{1}$ 6-epi-stephacidin A: ${ }^{1} \mathrm{H}$ NMR (400 MHz, $\left.\mathrm{CDCl}_{3}: \mathrm{CD}_{3} \mathrm{OD}, 20: 1\right) \delta 1.21$ (s, 3 H), $1.28(\mathrm{~s}, 3 \mathrm{H}), 1.41(\mathrm{~s}, 3 \mathrm{H}), 1.42(\mathrm{~s}, 3 \mathrm{H}), 1.81(\mathrm{dt}, J=7.4,14.6 \mathrm{~Hz}, 1 \mathrm{H}), 1.88-2.09$ (m, $4 \mathrm{H}), 2.24(\mathrm{dd}, J=4.1,9.9 \mathrm{~Hz}, 1 \mathrm{H}), 2.71(\mathrm{~m}, 1 \mathrm{H}), 2.82(\mathrm{~d}, J=17.9 \mathrm{~Hz}, 1 \mathrm{H}), 3.42-$ $3.52(\mathrm{~m}, 2 \mathrm{H}), 3.78(\mathrm{~d}, J=17.9 \mathrm{~Hz}, 1 \mathrm{H}), 5.62(\mathrm{~d}, J=9.7 \mathrm{~Hz}, 1 \mathrm{H}), 6.61(\mathrm{~d}, J=8.3 \mathrm{~Hz}, 1$ H), $6.64(\mathrm{~d}, J=9.7 \mathrm{~Hz}, 1 \mathrm{H}), 7.19(\mathrm{~d}, J=8.3 \mathrm{~Hz}, 1 \mathrm{H}), 8.54(\mathrm{~s}, 1 \mathrm{H}) ;{ }^{13} \mathrm{C}$ NMR $(100 \mathrm{MHz}$ $\mathrm{CDCl}_{3}: \mathrm{CD}_{3} \mathrm{OD}, 20:$ 1) $\delta 23.6,24.5,24.9,27.3,27.5,28.8,29.1,32.7,34.7,44.2,45.9$, $61.6,67.1,75.7,103.8,105.3,110.1,117.4,118.3,122.1,129.7,133.1,138.7,148.7$

${ }^{1}$ Greshock, T. J.; Grubbs, A. W.; Tsukamoto, S.; Williams, R. M. Angew. Chem. Int. Ed. 2007, 46, 2262. 
169.5, 173.3; IR (neat) 3291, 2917, 1693, $1666 \mathrm{~cm}^{-1}$; ESI/APCI-HRMS $\left(\mathrm{MH}^{+}\right)$calcd for $\mathrm{C}_{26} \mathrm{H}_{30} \mathrm{~N}_{3} \mathrm{O}_{3}$ 432.2282, found 432.2267 . 


\section{Improved Stephacidin A Synthesis}
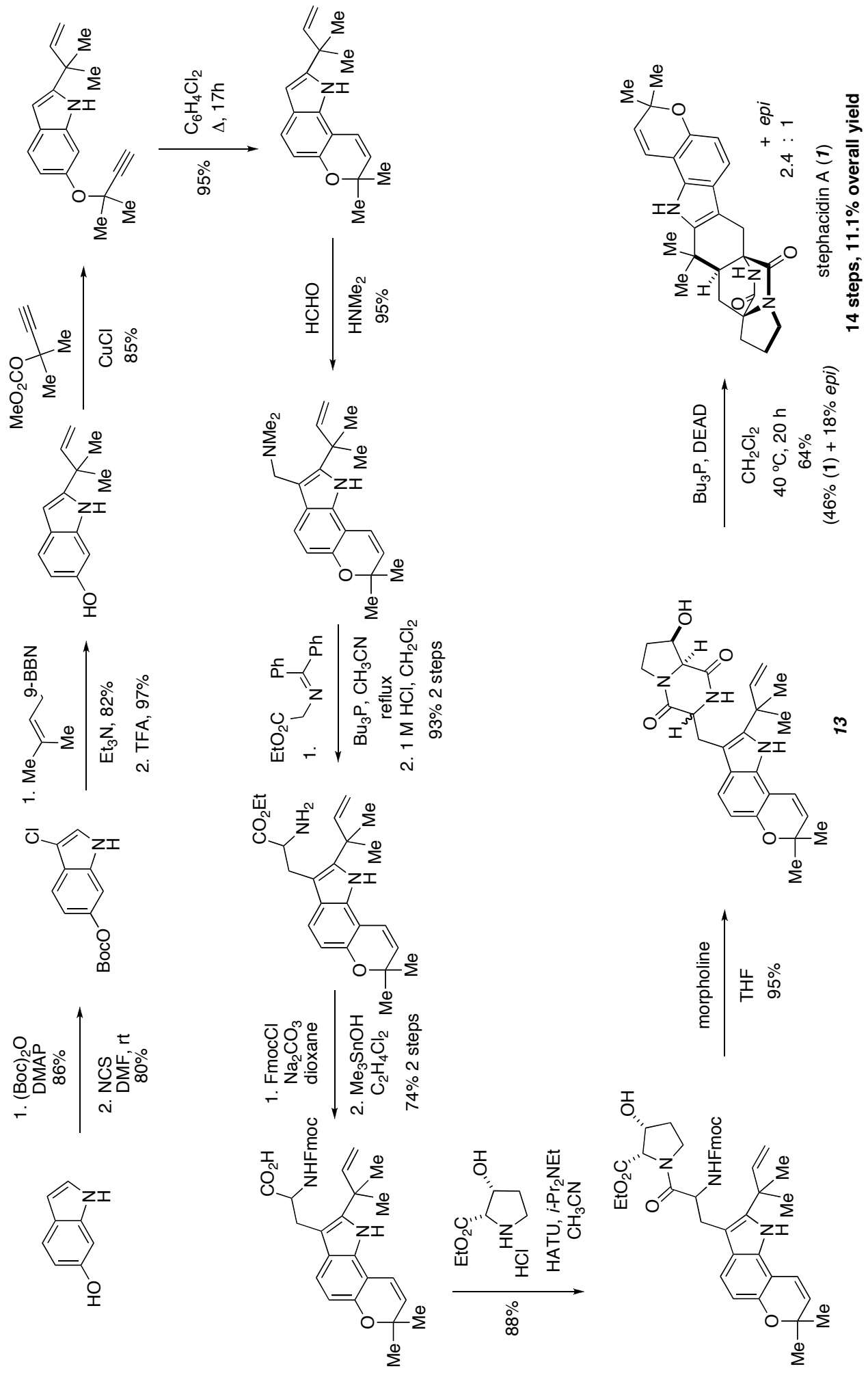


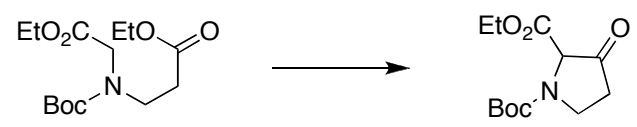

$\boldsymbol{N}$-Boc-3-ketoproline ethyl ester. To a solution of LIHMDS (1.0 M in THF, $7.91 \mathrm{~mL}$, $7.91 \mathrm{mmol})$ in THF $(60 \mathrm{~mL})$ at $-78{ }^{\circ} \mathrm{C}$ was added ethyl 3-(tert-butoxycarbonyl(2-ethoxy2-oxoethyl)-amino)propanoate ${ }^{2}$ in THF $(6 \mathrm{~mL})$ dropwise over $5 \mathrm{~min}$. The mixture was stirred at $-78{ }^{\circ} \mathrm{C}$ for $4 \mathrm{~h}$. The resulting solution was poured onto $1 \mathrm{M}$ aqueous $\mathrm{HCl}$ and extracted 3 times with ether. The combined extracts were washed 2 times with $10 \%$ aqueous $\mathrm{Na}_{2} \mathrm{CO}_{3}$, dried $\left(\mathrm{Na}_{2} \mathrm{SO}_{4}\right)$, and concentrated to afford $\mathrm{N}$-Boc-3-ketoproline ethyl ester as a colorless oil $(1.20 \mathrm{~g}, 71 \%)$; All spectral data was identical to that previously reported. $^{2}$

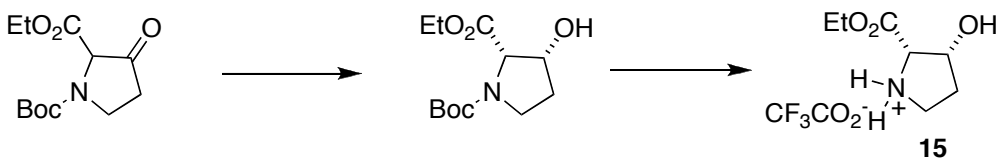

cis-3-Hydroxyproline ethyl ester trifluoroacetic acid salt (15). To a solution of $N$ Boc-3-ketoproline ethyl ester $(450 \mathrm{mg}, 1.75 \mathrm{mmol})$ in $\mathrm{MeOH}(9 \mathrm{~mL})$ at $0{ }^{\circ} \mathrm{C}$ was added $\mathrm{NaBH}_{4}(66 \mathrm{mg}, 1.75 \mathrm{mmol})$ in one portion. The mixture was stirred at $0{ }^{\circ} \mathrm{C}$ for $25 \mathrm{~min}$. The resulting solution was quenched with $1 \mathrm{M}$ aqueous $\mathrm{HCl}$ and extracted with ether. The combined extracts were dried $\left(\mathrm{Na}_{2} \mathrm{SO}_{4}\right)$ and concentrated to afford the crude cis- $N$ Boc-3-hydroxyproline ethyl ester along with a small amount of the trans diastereomer (424 mg, 93\%) as a mixture of amide rotamers, which was carried on without further

\footnotetext{
${ }^{2}$ Williams, R. M.; Cao, J.; Tsujishima, H.; Cox, R. J. J. Am. Chem. Soc. 2003, 125, 12172.
} 
purification. ${ }^{1} \mathrm{H}$ NMR $\left(300 \mathrm{MHz}, \mathrm{CDCl}_{3}\right) \delta 1.25(\mathrm{~m}, 3 \mathrm{H}), 1.37$ and $1.42(\mathrm{~s}, 9 \mathrm{H}), 1.91-$ $2.11(\mathrm{~m}, 2 \mathrm{H}), 2.80(\mathrm{br} \mathrm{s}, 1 \mathrm{H}), 3.42(\mathrm{~m}, 1 \mathrm{H}), 3.60(\mathrm{~m}, 1 \mathrm{H}), 4.10-4.39(\mathrm{~m}, 3 \mathrm{H}), 4.57$ $(\mathrm{m}, 1 \mathrm{H})$. To a solution of the crude alcohol $(276 \mathrm{mg}, 1.06 \mathrm{mmol})$ in $\mathrm{CH}_{2} \mathrm{Cl}_{2}(5 \mathrm{~mL})$ at 0 ${ }^{\circ} \mathrm{C}$ was added TFA ( $\left.1 \mathrm{~mL}\right)$. The mixture was warmed to $\mathrm{rt}$ and stirred $3 \mathrm{~h}$. The resulting solution was concentrated directly to afford the crude amine TFA salt 15 , which was used directly in the next reaction without further purification.
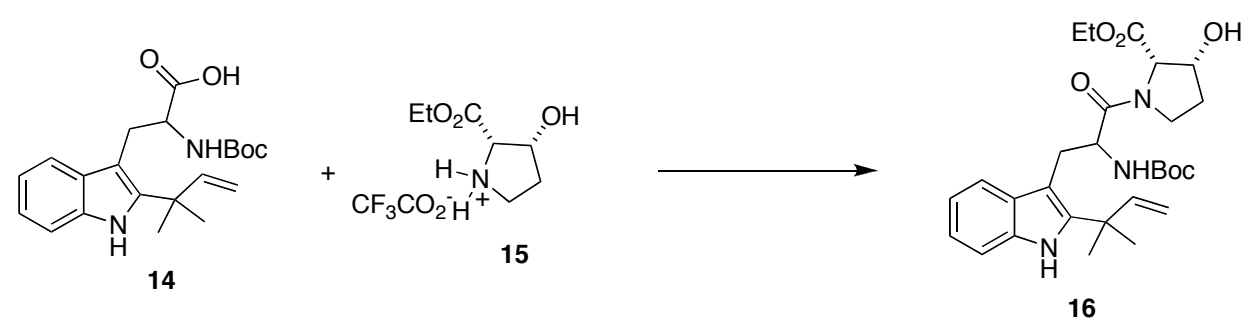

Peptide (16). To a solution of acid $14^{3}$ (330 mg, $\left.0.89 \mathrm{mmol}\right)$ in $\mathrm{CH}_{3} \mathrm{CN}(4.5 \mathrm{~mL})$ was added HATU (505 mg, $1.33 \mathrm{mmol}$ ), $\mathbb{P P r}_{2} \mathrm{NEt}(617 \mu \mathrm{L}, 3.54 \mathrm{mmol}$ ), and cis-3hydroxyproline ethyl ester $\mathbf{1 5}$ successively at $\mathrm{rt}$. The mixture was stirred at $\mathrm{rt}$ for $3 \mathrm{~h}$. The resulting solution was quenched with $2 \mathrm{M}$ aqueous $\mathrm{HCl}$ and extracted with $\mathrm{CH}_{2} \mathrm{Cl}_{2}$. The combined extracts were dried $\left(\mathrm{Na}_{2} \mathrm{SO}_{4}\right)$ and concentrated. Purification by silica gel chromatography (ethyl acetate : hexane, $3: 1$ ) afforded amide 16 as a mixture of diastereomers and amide rotamers (440 mg, 97\%); $\left.{ }^{1} \mathrm{H} \mathrm{NMR} \mathrm{(400} \mathrm{MHz,} \mathrm{CDCl}_{3}\right) \delta 1.21$ and $1.25(\mathrm{t}, J=7.1 \mathrm{~Hz}, 3 \mathrm{H}), 1.41(\mathrm{~s}, 9 \mathrm{H}), 1.58(\mathrm{~s}, 3 \mathrm{H}), 1.60(\mathrm{~s}, 3 \mathrm{H}), 2.12-2.21(\mathrm{~m}, 2$ H), $3.00-3.40(\mathrm{~m}, 4 \mathrm{H}), 3.59$ and $3.90(\mathrm{~m}, 1 \mathrm{H}), 4.06(\mathrm{~m}, 1 \mathrm{H}), 4.14$ and $4.21(\mathrm{q}, J=7.1$

${ }^{3}$ Stocking, E. M.; Sanz-Cervera, J. F.; Williams, R. M. J. Am. Chem. Soc. 2000, 122, 1675. 
$\mathrm{Hz}, 2 \mathrm{H}), 4.61(\mathrm{~m}, 1 \mathrm{H}), 5.16(\mathrm{~d}, J=10.6 \mathrm{~Hz}, 1 \mathrm{H}), 5.20(\mathrm{~d}, J=17.6 \mathrm{~Hz}, 1 \mathrm{H}), 5.58$ and 5.60 (br s, $1 \mathrm{H}), 6.09$ (dd, J = 10.6, $17.6 \mathrm{~Hz}, 1 \mathrm{H}), 6.95-7.11(\mathrm{~m}, 2 \mathrm{H}), 7.21(\mathrm{~m}, 1 \mathrm{H})$, $7.40(\mathrm{~m}, 1 \mathrm{H}), 8.00$ and $8.12(\mathrm{~s}, 1 \mathrm{H}) ;{ }^{13} \mathrm{C} \mathrm{NMR}\left(100 \mathrm{MHz}, \mathrm{CDCl}_{3}\right) \delta$ 27.4, 27.9, 28.3, $28.5,30.5,30.8,31.1,32.4,33.8,39.2,44.0,44.3,45.4,52.8,53.4,61.6,62.1,62.9$, $63.9,65.1,70.0,70.3,79.7,106.1,110.5,112.7,118.7,119.5,121.9,129.7,133.8$ 140.8, 145.4, 154.6, 155.1, 167.8, 169.3, 171.9; IR (neat) 3383, 2977, 1740, 1694, 1635 $\mathrm{cm}^{-1}$; ESI/APCI-HRMS $\left(\mathrm{MH}^{+}\right)$calcd for $\mathrm{C}_{28} \mathrm{H}_{40} \mathrm{~N}_{3} \mathrm{O}_{6}$ 514.2912, found 514.2899.
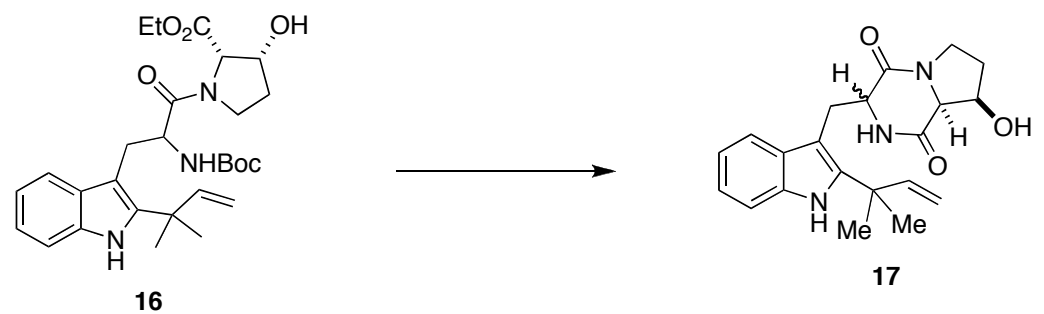

Alcohol (17). To a solution of amide $16(439 \mathrm{mg}, 0.85 \mathrm{mmol})$ in $\mathrm{CH}_{2} \mathrm{Cl}_{2}(17 \mathrm{~mL})$ at $0{ }^{\circ} \mathrm{C}$ was added TFA $(1.7 \mathrm{~mL})$. The mixture was warmed to $\mathrm{rt}$ and stirred $2 \mathrm{~h}$. The resulting solution was quenched slowly with saturated aqueous $\mathrm{NaHCO}_{3}$ and extracted with $\mathrm{CH}_{2} \mathrm{Cl}_{2}$. The combined extracts were dried $\left(\mathrm{Na}_{2} \mathrm{SO}_{4}\right)$ and concentrated to afford the crude primary amine. To a solution of the crude primary amine in toluene (17 mL) was added 2-hydroxypyridine ( $16.3 \mathrm{mg}, 0.17 \mathrm{mmol})$. The mixture was heated to reflux for 16 $\mathrm{h}$ and then concentrated. The crude residue was diluted with $\mathrm{CH}_{2} \mathrm{Cl}_{2}$, washed twice with $1 \mathrm{M} \mathrm{HCl}$, dried $\left(\mathrm{Na}_{2} \mathrm{SO}_{4}\right)$ and concentrated to afford alcohol 17 as an inseparable mixture of diastereomers (292 mg, 93\%); ${ }^{1} \mathrm{H}$ NMR (400 MHz, $\left.\mathrm{CDCl}_{3}\right) \delta 1.48(\mathrm{~s}, 3 \mathrm{H})$, $1.52(\mathrm{~s}, 3 \mathrm{H}), 1.92-2.18(\mathrm{~m}, 2 \mathrm{H}), 3.21(\mathrm{~m}, 1 \mathrm{H}), 3.48(\mathrm{~m}, 1 \mathrm{H}), 3.62-3.95(\mathrm{~m}, 3 \mathrm{H})$, 
$4.20(\mathrm{~m}, 1 \mathrm{H}), 4.58(\mathrm{~m}, 1 \mathrm{H}), 5.09-5.18(\mathrm{~m}, 2 \mathrm{H}), 5.90$ and $6.40(\mathrm{~s}, 1 \mathrm{H}), 6.10(\mathrm{~m}, 1 \mathrm{H})$, $7.00-7.35(\mathrm{~m}, 3 \mathrm{H}), 7.47(\mathrm{~m}, 1 \mathrm{H}), 8.24$ and $8.33(\mathrm{~s}, 1 \mathrm{H}) ;{ }^{13} \mathrm{C} \mathrm{NMR}\left(100 \mathrm{MHz}, \mathrm{CDCl}_{3}\right) \delta$ $27.8,28.0,29.8,30.0,30.4,33.5,39.0,44.0,44.2,45.2,54.9,58.4,61.8,63.9,64.6$ $65.1,69.8,70.8,104.4,104.9,110.6,111.0,112.0,112.8,117.9,118.5,119.7,120.1$ $121.8,122.1,125.4,128.3,129.0,134.3,134.5,141.8,145.6,146.0,165.8,166.4$ 167.4; IR (neat) 3358, 2969, 1742, $1655 \mathrm{~cm}^{-1}$; ESI/APCI-HRMS $\left(\mathrm{MH}^{+}\right)$calcd for $\mathrm{C}_{21} \mathrm{H}_{26} \mathrm{~N}_{3} \mathrm{O}_{3} 368.1969$, found 368.1957.

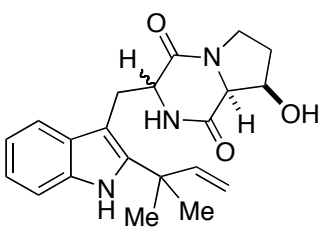

17

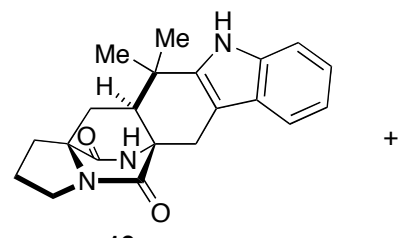

19

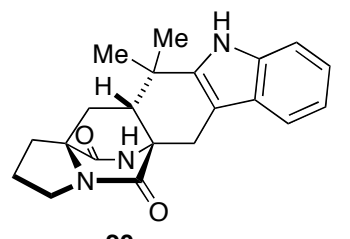

20

Cycloadducts (19 and 20). To a solution of alcohols 17 (100 mg, $0.27 \mathrm{mmol})$ in $\mathrm{CH}_{2} \mathrm{Cl}_{2}$ $(27 \mathrm{~mL})$ at rt was added DEAD $(257 \mu \mathrm{L}, 1.63 \mathrm{mmol})$. The mixture was stirred at rt for 5 min and $\mathrm{PBu}_{3}(408 \mu \mathrm{L}, 1.63 \mathrm{mmol})$ was then added. The solution was heated to $40^{\circ} \mathrm{C}$ for $20 \mathrm{~h}$. The resulting mixture was concentrated. Purification by silica gel chromatography (ethyl acetate : hexane, $1: 1$, then methanol : $\mathrm{CH}_{2} \mathrm{Cl}_{2}, 5: 95$ ) afforded cycloadducts 19 and 20 as a $2.1: 1$ crude mixture of diastereomers, respectively. This mixture was further separated by preparative TLC $\left(\mathrm{MeOH}: \mathrm{CH}_{2} \mathrm{Cl}_{2}, 3: 97\right)$ to afford syn cycloadduct 19 as a white solid (43.6 $\mathrm{mg}, 46 \%)$ along with anti cycloadduct 20 as a 
white solid (20.1 mg, 21\%); Both cycloadducts $19^{4}$ and $20^{5}$ have been previously synthesized. All data was identical to that previously reported.

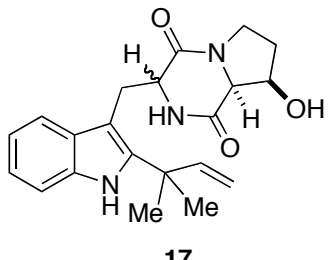

17

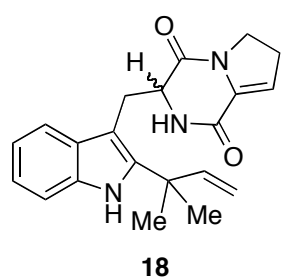

18

Enamide (18). To a solution of alcohols $17(120 \mathrm{mg}, 0.33 \mathrm{mmol})$ in $\mathrm{CH}_{2} \mathrm{Cl}_{2}(6.5 \mathrm{~mL})$ at rt was added DEAD ( $40 \%$ wt. in toluene, $446 \mu \mathrm{L}, 0.98 \mathrm{mmol})$. The mixture was stirred at rt for 5 min and $\mathrm{PBu}_{3}(245 \mu \mathrm{L}, 0.98 \mathrm{mmol})$ was then added. The solution was stirred at rt for $3 \mathrm{~h}$. The resulting mixture was then concentrated. Purification by silica gel chromatography (ethyl acetate : hexane, $2: 3$ to $1: 0$ ) afforded enamide 18 as a white foam (98.2 mg, 86\%); ${ }^{1} \mathrm{H}$ NMR (400 MHz, $\left.\mathrm{CDCl}_{3}\right) \delta 1.52(\mathrm{~s}, 3 \mathrm{H}), 1.53(\mathrm{~s}, 3 \mathrm{H}), 2.76(\mathrm{~m}$, $2 \mathrm{H}), 3.22(\mathrm{dd}, J=11.3,14.5 \mathrm{~Hz}, 1 \mathrm{H}), 3.70(\mathrm{dd}, J=3.5,14.5 \mathrm{~Hz}, 1 \mathrm{H}), 4.05(\mathrm{~m}, 2 \mathrm{H})$, $4.50(\mathrm{br} \mathrm{d}, J=10.3 \mathrm{~Hz}, 1 \mathrm{H}), 5.12(\mathrm{~d}, J=10.5 \mathrm{~Hz}, 1 \mathrm{H}), 5.14(\mathrm{~d}, J=17.5 \mathrm{~Hz}, 1 \mathrm{H}), 5.73$ (s, $1 \mathrm{H}), 6.10$ (dd, J = 10.5, 17.5 Hz, $1 \mathrm{H}), 6.13(\mathrm{t}, J=2.9 \mathrm{~Hz}, 1 \mathrm{H}), 7.08(\mathrm{t}, J=7.7 \mathrm{~Hz}, 1$ H), $7.14(\mathrm{t}, J=7.7 \mathrm{~Hz}, 1 \mathrm{H}), 7.30(\mathrm{~d}, J=7.7 \mathrm{~Hz}, 1 \mathrm{H}), 7.50(\mathrm{~d}, J=7.7 \mathrm{~Hz}, 1 \mathrm{H}), 8.39(\mathrm{~s}$, $1 \mathrm{H}) ;{ }^{13} \mathrm{C}$ NMR $\left(100 \mathrm{MHz}, \mathrm{CDCl}_{3}\right) \delta 27.8,27.9,28.0,30.9,39.1,45.6,57.5,104.4,110.9$, 112.3, 118.2, 119.0, 120.0, 122.0, 128.8, 133.1, 134.4, 141.8, 145.8, 156.5, 162.6; IR

${ }^{4}$ (a) Williams, R. M.; Glinka, T.; Kwast, E.; Coffman, H.; Stille, J. K. J. Am. Chem. Soc. 1990, 112, 808. (b) Jin, S.; Wessig, P.; Liebscher, J. J. Org. Chem. 2001, 66, 3984. (c) Baran, P. S.; Hafensteiner, B. D.; Ambhaikar, N. B.; Guerrero, C. A.; Gallagher, J. D. J. Am. Chem. Soc. 2006, 128, 8678.

${ }^{5}$ Adams, L. A.; Valente, M. W. N.; Williams, R. M. Tetrahedron 2006, 62, 5195. 
(neat) $3341,2967,1677,1644 \mathrm{~cm}^{-1}$; ESI/APCI-HRMS $\left(\mathrm{MH}^{+}\right)$calcd for $\mathrm{C}_{21} \mathrm{H}_{24} \mathrm{~N}_{3} \mathrm{O}_{2}$ 350.1863 , found 350.1842 .

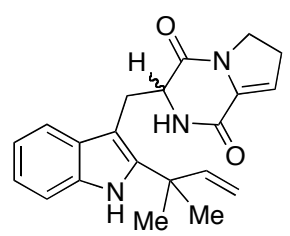

18

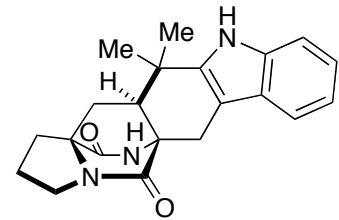

19

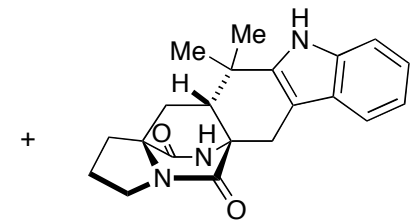

20

Cycloadducts (19 and 20). To a solution of enamide 18 (92 mg, $0.26 \mathrm{mmol}$ ) in $\mathrm{MeOH}$ $(20 \mathrm{~mL})$ at $0{ }^{\circ} \mathrm{C}$ was added $20 \%$ aqueous $\mathrm{KOH}(5 \mathrm{~mL})$. The mixture was slowly warmed to rt over $1 \mathrm{~h}$ and stirred for $18 \mathrm{~h}$. The resulting solution was quenched with saturated aqueous $\mathrm{NH}_{4} \mathrm{Cl}$ and extracted with $\mathrm{CH}_{2} \mathrm{Cl}_{2}$. The combined extracts were dried $\left(\mathrm{Na}_{2} \mathrm{SO}_{4}\right)$ and concentrated. Purification by preparative TLC $\left(\mathrm{MeOH}: \mathrm{CH}_{2} \mathrm{Cl}_{2}, 3: 97\right)$ afforded syn cycloadduct 19 as a white solid (55.2 $\mathrm{mg}, 60 \%$ ) along with anti cycloadduct 20 as a white solid (26.9 mg, 29\%).

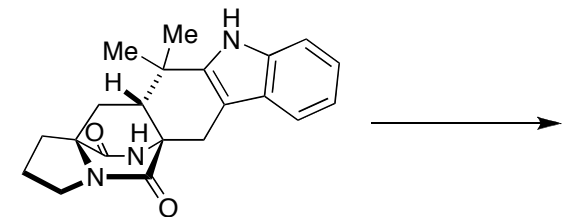

20

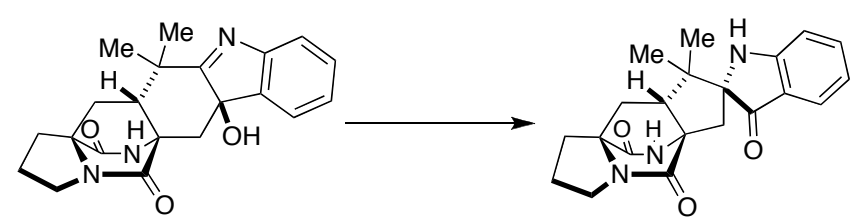

(士)-brevianamide B (9)

Brevianamide B (9). To a solution of indole $20(10.0 \mathrm{mg}, 0.029 \mathrm{mmol})$ in THF $(1 \mathrm{~mL})$ at rt was added $m$ CPBA $(6.4 \mathrm{mg}, 0.037 \mathrm{mmol})$. The mixture was stirred at $\mathrm{rt}$ for $1.5 \mathrm{~h}$. The resulting solution was quenched with two drops of dimethyl sulfide and 
concentrated to dryness to afford the crude 3-hydroxyindolinene, which was used without further purification. To a solution of the crude 3-hydroxyindolinene in $\mathrm{MeOH}$ (1 $\mathrm{mL}$ ) was added $0.5 \mathrm{M}$ aqueous $\mathrm{NaOH}(2 \mathrm{~mL})$. The mixture was stirred at $\mathrm{rt}$ for $16 \mathrm{~h}$ and then warmed to $70^{\circ} \mathrm{C}$ for $2 \mathrm{~h}$. The resulting solution was cooled to rt, quenched with 1 $\mathrm{M}$ aqueous $\mathrm{HCl}$, and extracted with $\mathrm{CH}_{2} \mathrm{Cl}_{2}$. The combined extracts were dried $\left(\mathrm{Na}_{2} \mathrm{SO}_{4}\right)$ and concentrated. Purification by preparative TLC (MeOH : $\left.\mathrm{CH}_{2} \mathrm{Cl}_{2}, 5: 95\right)$ afforded brevianamide B (9) as a yellow film (4.7 mg, 45\%); All spectral data was identical to the natural sample as well as those synthetic samples previously prepared in our group. ${ }^{5}$ See attached ${ }^{1} \mathrm{H}$ NMR spectrum.

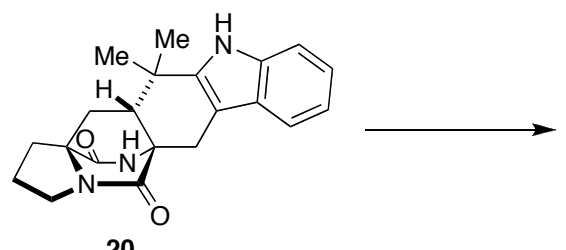

20

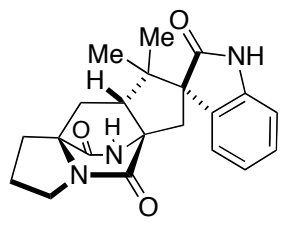

23

Oxindole (23). To a solution of indole $20(16 \mathrm{mg}, 0.046 \mathrm{mmol})$ in $\mathrm{CH}_{2} \mathrm{Cl}_{2}(1.8 \mathrm{~mL})$ at rt was added oxaziridine 22 (33 mg, $0.14 \mathrm{mmol}$ ). The mixture was stirred at $\mathrm{rt}$ for $16 \mathrm{~h}$. The resulting solution was placed directly on a column of silica gel and eluted with $\mathrm{CH}_{2} \mathrm{Cl}_{2}$, followed by $\mathrm{MeOH}: \mathrm{CH}_{2} \mathrm{Cl}_{2}(5: 95)$ to afford oxindole 23 as a thin film (11.8 $\mathrm{mg}$, 71\%); ${ }^{1} \mathrm{H}$ NMR (400 MHz, $\left.\mathrm{CDCl}_{3}\right) \delta 1.33(\mathrm{~s}, 3 \mathrm{H}), 1.45$ (s, $\left.3 \mathrm{H}\right), 1.84$ (dt, J = 7.3, 13.1 $\mathrm{Hz}, 1 \mathrm{H}), 1.96-2.10(\mathrm{~m}, 5 \mathrm{H}), 2.75(\mathrm{dt}, J=6.7,13.1 \mathrm{~Hz}, 1 \mathrm{H}), 2.82(\mathrm{~d}, J=15.8 \mathrm{~Hz}, 1$ H), $3.42(\mathrm{t}, J=6.7 \mathrm{~Hz}, 2 \mathrm{H}), 3.49(\mathrm{~m}, 1 \mathrm{H}), 5.30(\mathrm{~s}, 1 \mathrm{H}), 7.13(\mathrm{~s}, 1 \mathrm{H}), 7.23(\mathrm{t}, J=7.5$ $\mathrm{Hz}, 1 \mathrm{H}), 7.37(\mathrm{t}, J=7.5 \mathrm{~Hz}, 1 \mathrm{H}), 7.43(\mathrm{~d}, J=7.5 \mathrm{~Hz}, 1 \mathrm{H}), 7.53(\mathrm{~d}, J=7.5 \mathrm{~Hz}, 1 \mathrm{H}) ;{ }^{13} \mathrm{C}$ 
$\operatorname{NMR}\left(100 \mathrm{MHz}, \mathrm{CDCl}_{3}\right) \delta 20.2,24.5,27.5,29.2,32.7,38.0,40.5,44.2,50.5,62.1,67.3$ $82.5,121.3,122.4,126.7,130.3,140.5,152.1,168.4,172.5,188.2$; IR (neat) 3365 , 2968, $1688 \mathrm{~cm}^{-1}$; ESI/APCI-HRMS $\left(\mathrm{MH}^{+}\right)$calcd for $\mathrm{C}_{21} \mathrm{H}_{24} \mathrm{~N}_{3} \mathrm{O}_{3}$ 366.1812, found 366.1800. 


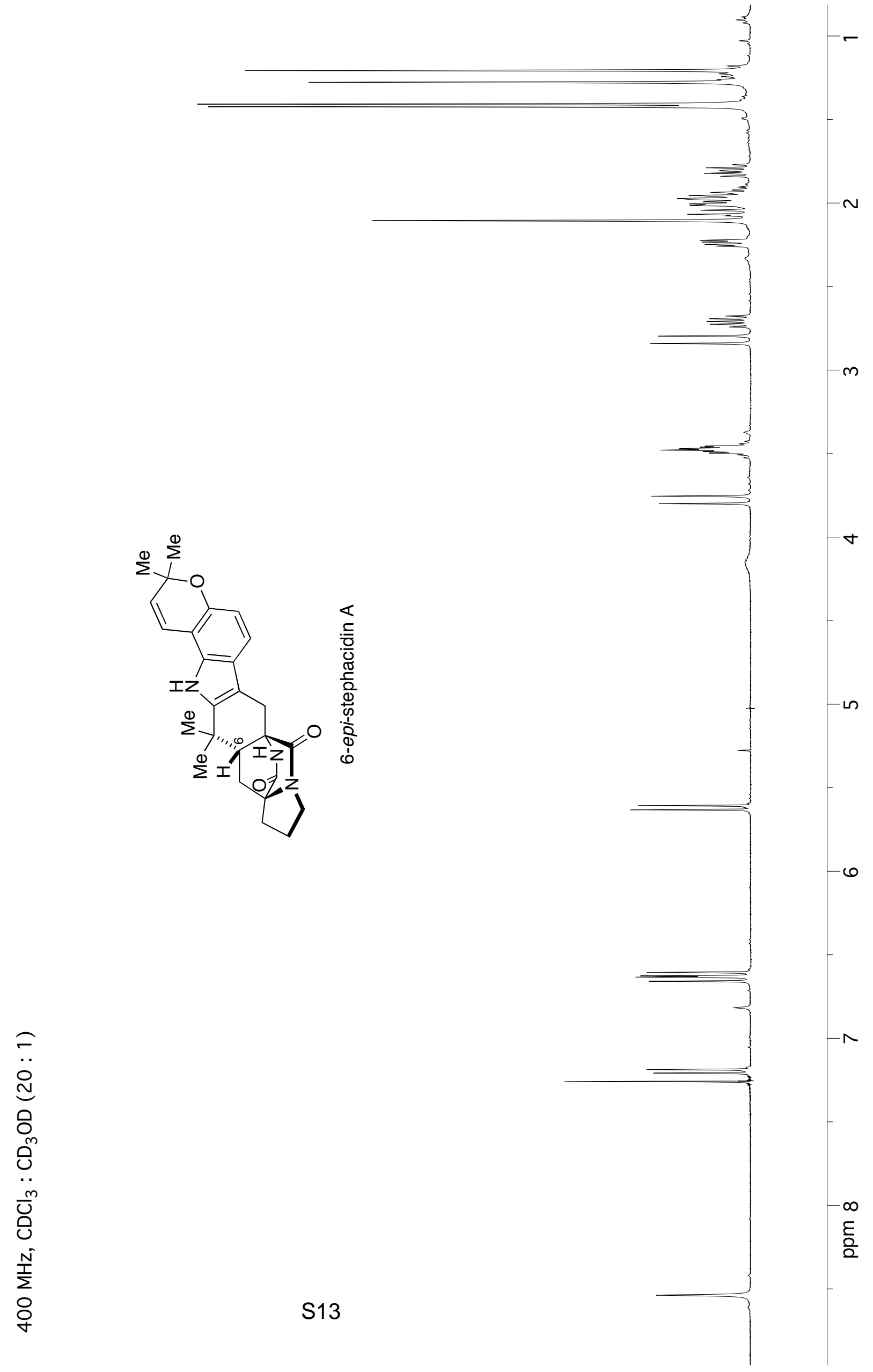


ع8S. $\varepsilon ट$

乙 IS"tट

128
$\rightarrow 92$

$86 t^{\circ} \angle 2$

808.82

S2l.62

$889^{\circ} 2 \varepsilon$

$\angle \varepsilon Z^{\circ} \nabla t$

$\varepsilon \angle 8^{\circ} \mathrm{S} t$

$809^{*} 19$

ยヤ L" $\angle 9$

$0 \angle 9^{\circ} S \angle$

Z6L'EOL
ELZ'SOL

92l'OLL

L8 $\varepsilon^{\circ} \angle L L$

टLEं8LL

9 L L'て2 L

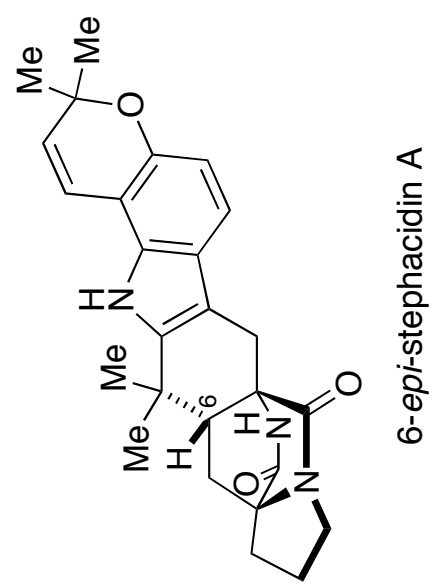

82L'62L

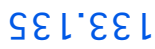

SOL'8EL

LZL'8tL

$\widetilde{\tau}$

음

ㅇ

巳

음

$\angle \forall S^{\circ} 69 \mathrm{~L}$

$\left\llcorner\vdash \varepsilon^{\cdot} \varepsilon\llcorner\mathrm{L}\right.$

$\stackrel{N}{N}$

ㄴ

으

우

운

-

웃

$\infty$

움

음

으

-

$\stackrel{ }{\sim}$

$\stackrel{\text { m }}{\sim}$

움

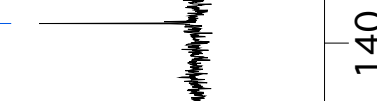

옴

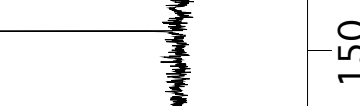

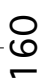

-
$-\infty$
$-\infty$
$-\infty$
$-\infty$
$-\infty$ 


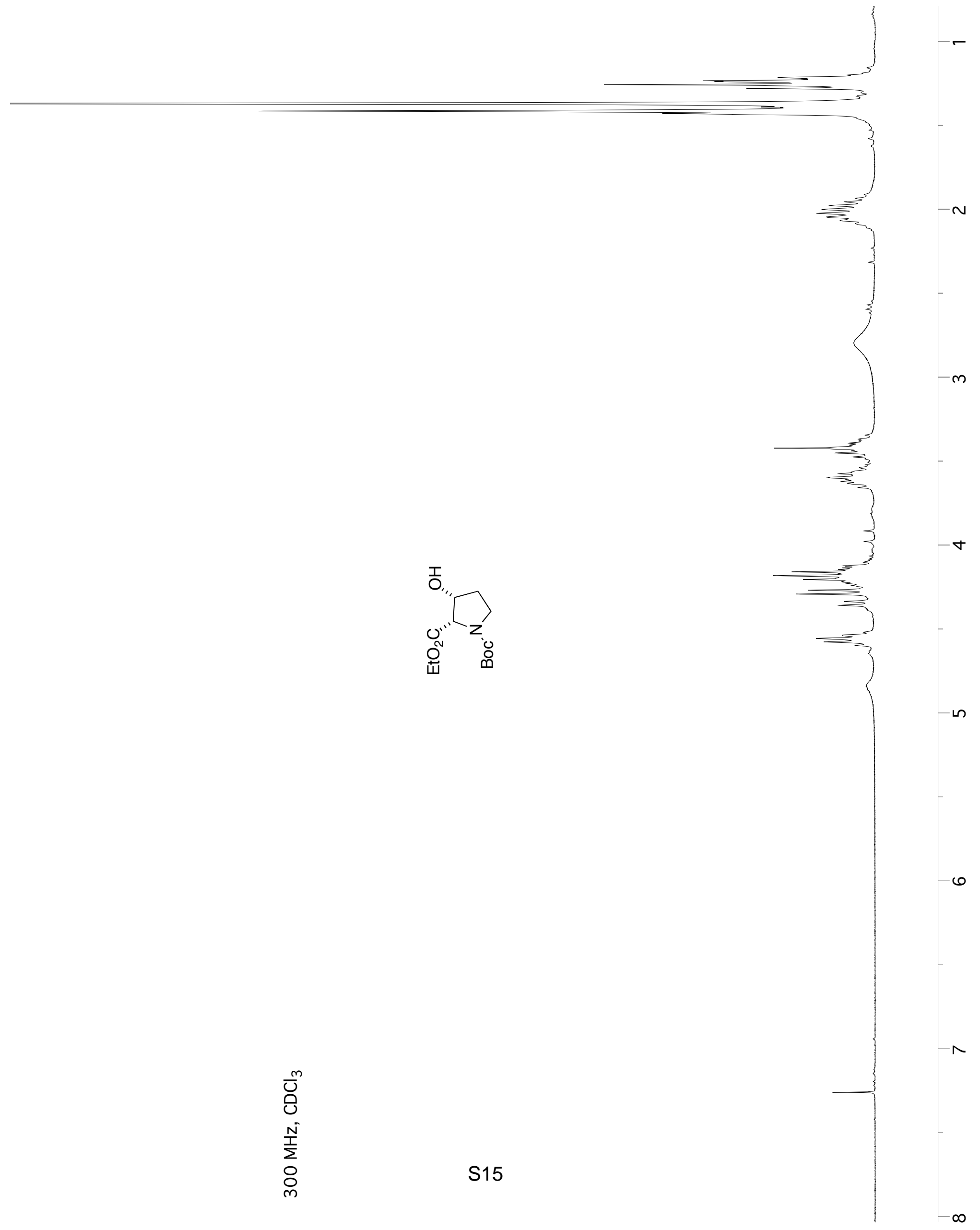




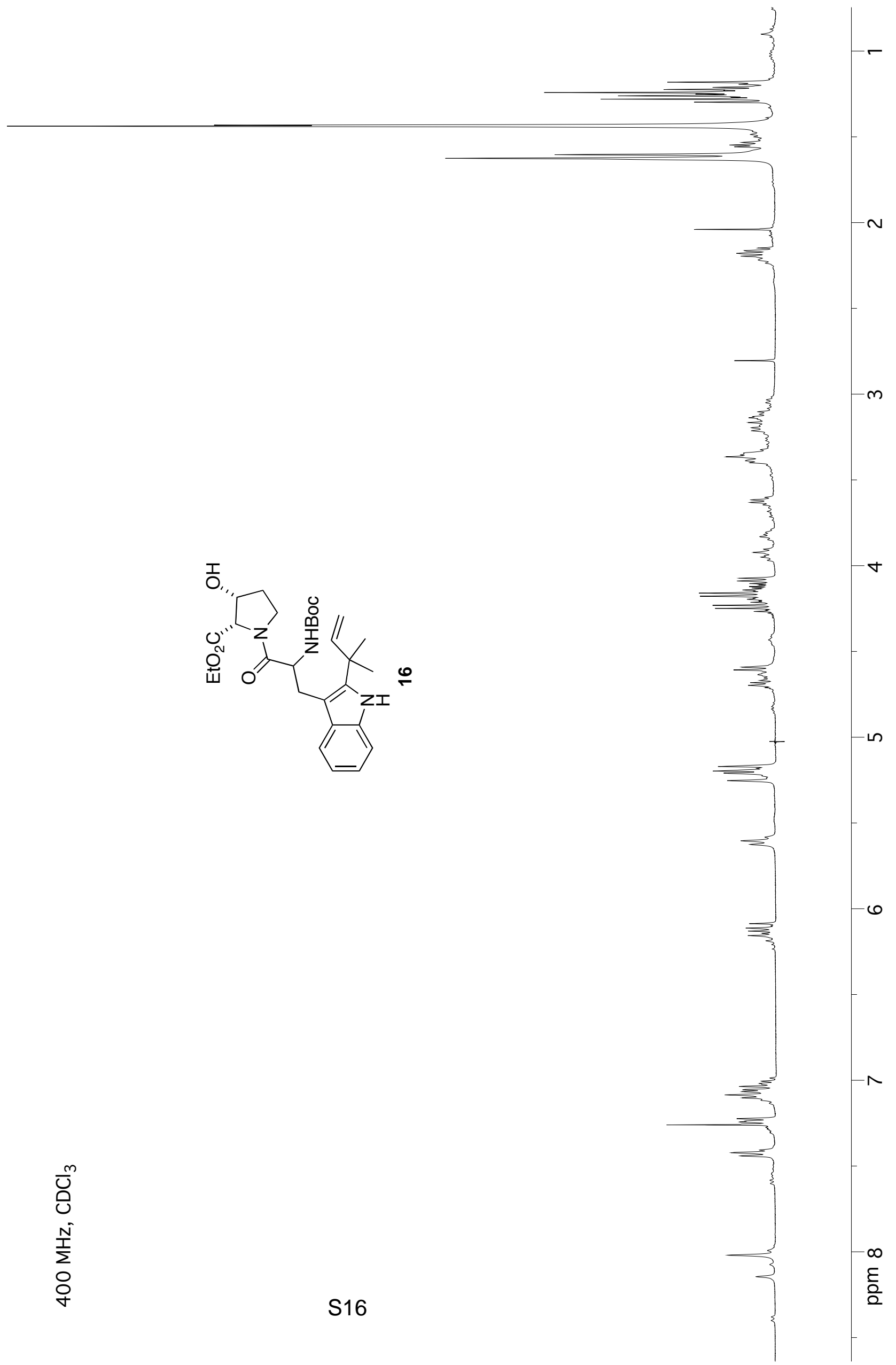


SOt" $\angle 2$

$\angle 06^{\circ} \angle 2$

St2.8Z

9ES'82

$S \angle D^{\circ} 0 \varepsilon$

8SL"OE

$080^{\circ} L$

$9 \varepsilon \nabla^{\circ} 乙 \varepsilon$

ᄂ $8{ }^{\prime} \varepsilon \varepsilon$

L I $2.6 \varepsilon$

OEO" $\nabla t$

$0 乙 \varepsilon " \nabla t$

$6 \angle \varepsilon^{*} S \triangleright$

$\varepsilon 6 L$ ' 2

$\neg \angle \mathcal{E}^{\circ} \varepsilon S$

2SS: 19

2 70.29

668.29

888. 29

SEL'S9

$6 \mathrm{LO}^{\circ} \mathrm{OL}$

SSZ"OL

$\varepsilon 89^{\circ} 6 L$

9S0.9OL

\&87"0 L L

$8 \varepsilon L^{\prime} Z L$

02L'8L L

925.6LL

ᄂ696乙 L

$\angle 08^{\circ} \varepsilon \varepsilon\llcorner$

66L'0†L

09ع'StL

S9S"tSL
bLO"SSL

98L" $\angle 9 L$

L82.69 L

$8 \varepsilon 6^{\circ} \mathrm{L} L \mathrm{~L}$

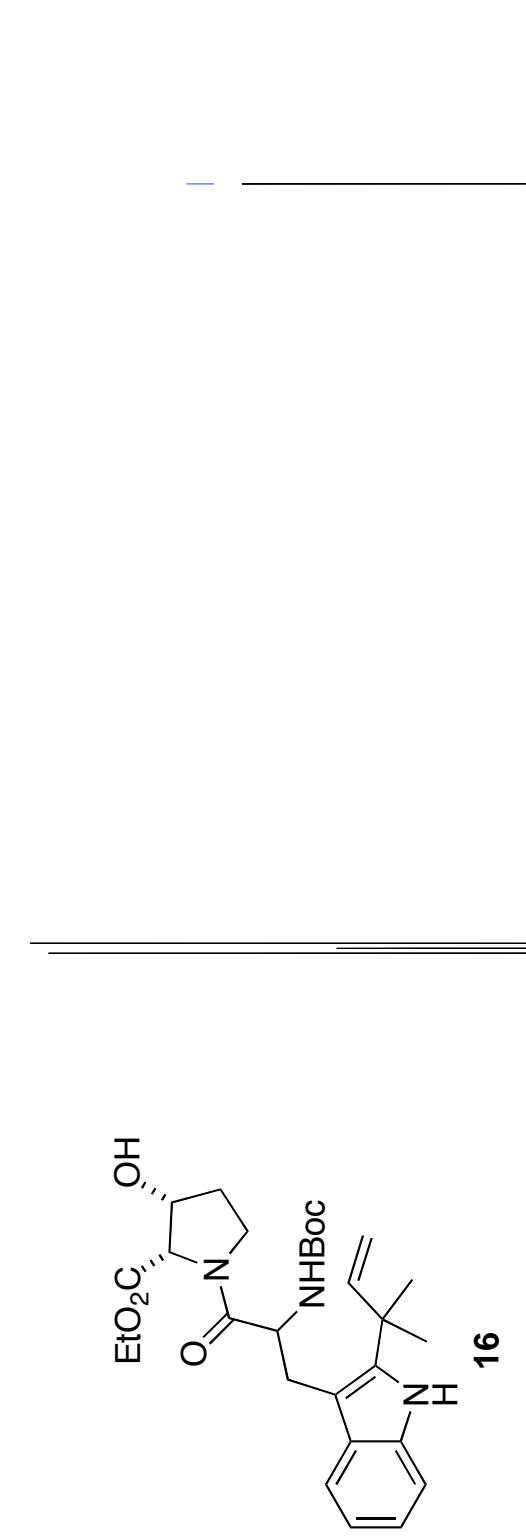

- ํ

이

우

운

8

-

웅

응

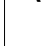

음

$-$

$\stackrel{-}{-}$

음 $-$

옹

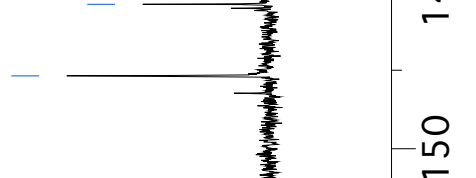

눈

$-8$

ปூ

N

응 


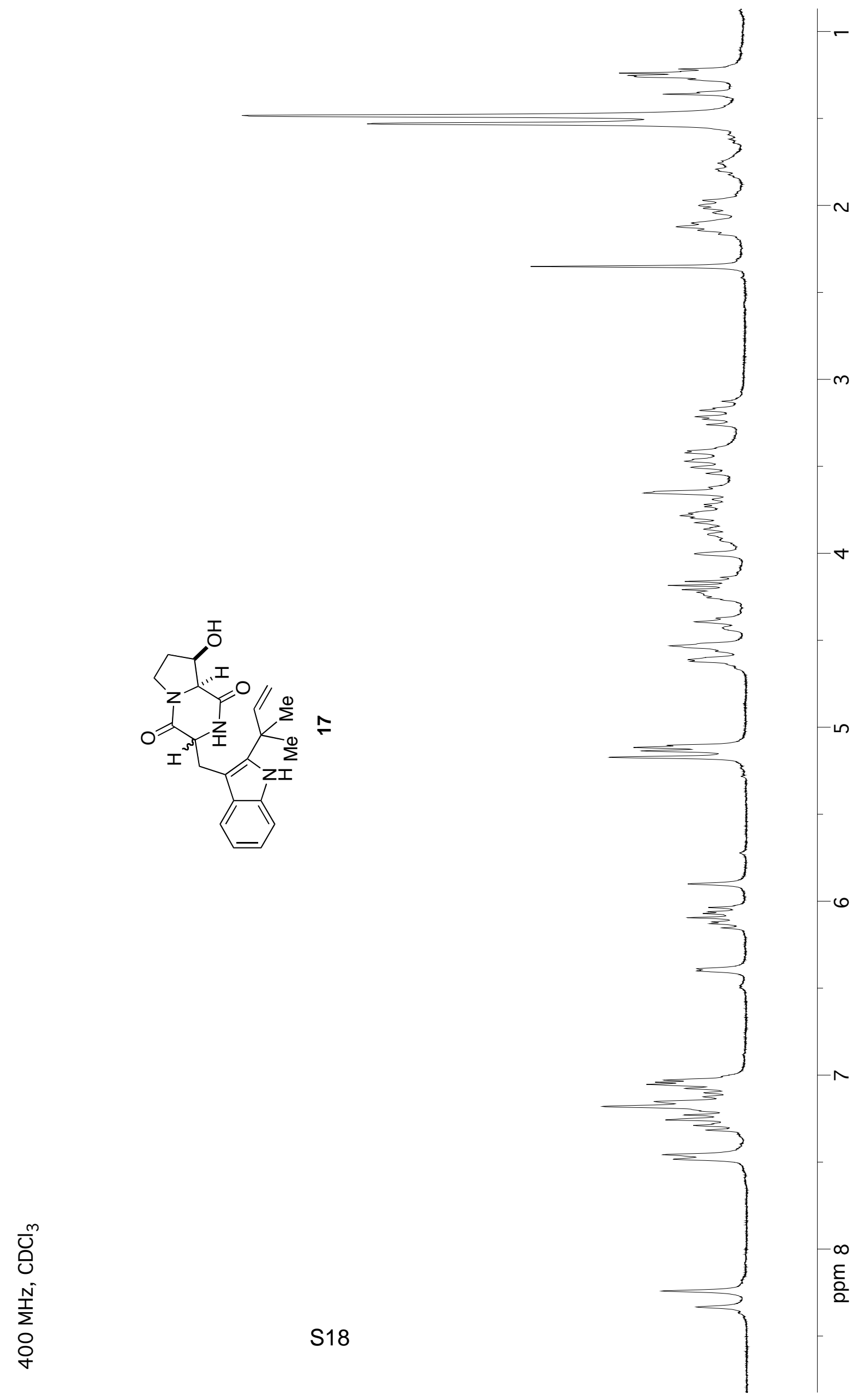


28L" $\angle 2$

818.62

686.62

$\neg 6 \mathcal{E}^{\circ} 0 \varepsilon$

ธES 'E

$6 \varepsilon 0^{\circ} 6 \varepsilon$

$9 ヤ 0 " \nabla t$

己Lでt

\section{$\angle S 6^{\circ} \supset S$}

¿SE" $8 S$

86L" 19

606.'99

$8 \angle S^{\circ} \rightarrow 9$

†90" 9

L28.69

$\angle 08^{\circ} 0 \angle$

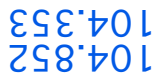
685. O L L $\rightarrow \varepsilon 0^{\circ} L L$ E86" L L 828" ' L L $\angle S 8^{\circ} \angle L L$ L $\angle t^{\circ} 8 \mathrm{~L} \mathrm{~L}$ $269^{\circ} 6 \mathrm{~L} \mathrm{~L}$ $\angle \angle O^{\circ} \mathrm{OZ} \mathrm{L}$ 9SLIZL

El l. Zl

ESE'Sट

282.82

$\rightarrow 60^{\circ} 62 \mathrm{~L}$

$S \angle Z^{\circ} \nabla \varepsilon$

9St"†

LL9"LL

88.'st L

966"St L

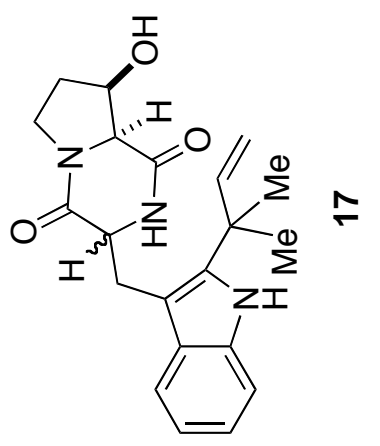

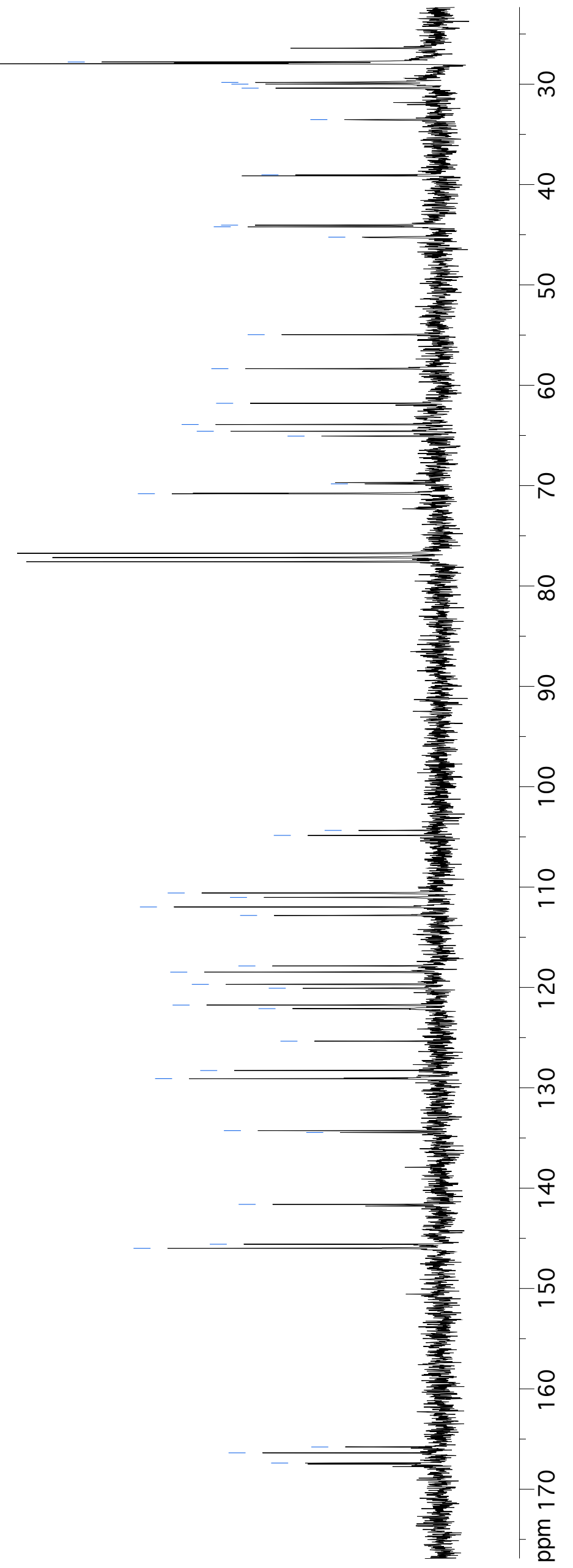




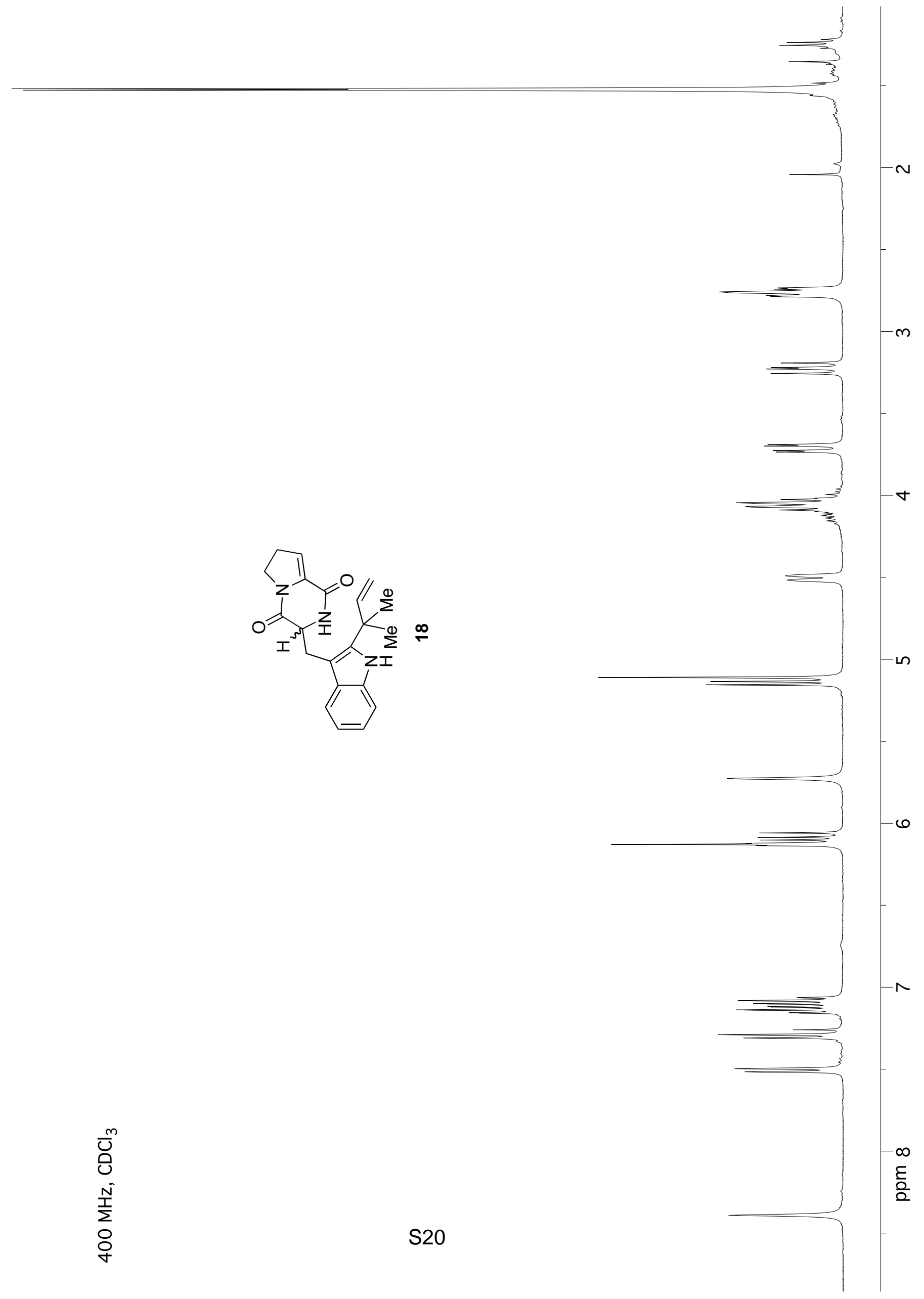


$8 \rightarrow 8^{\circ}<2$

$0 \rightarrow 6 . \angle 2$

$686^{\circ} \mathrm{LC}$

OS $8^{\circ} 0 \varepsilon$

$080^{\circ} 6 \varepsilon$

E\&9"St

$\neg 8 \nabla^{\circ} \angle S$

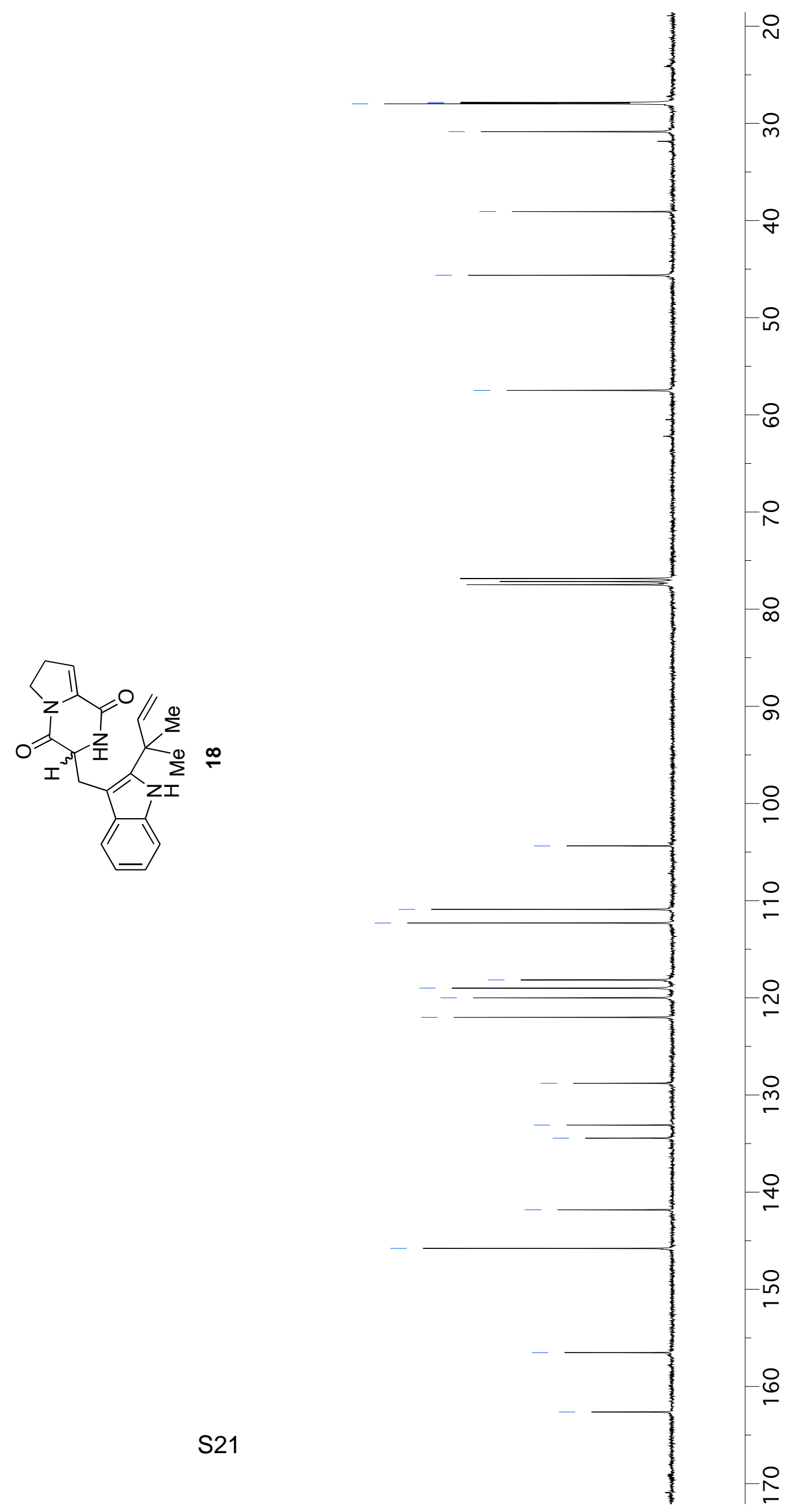




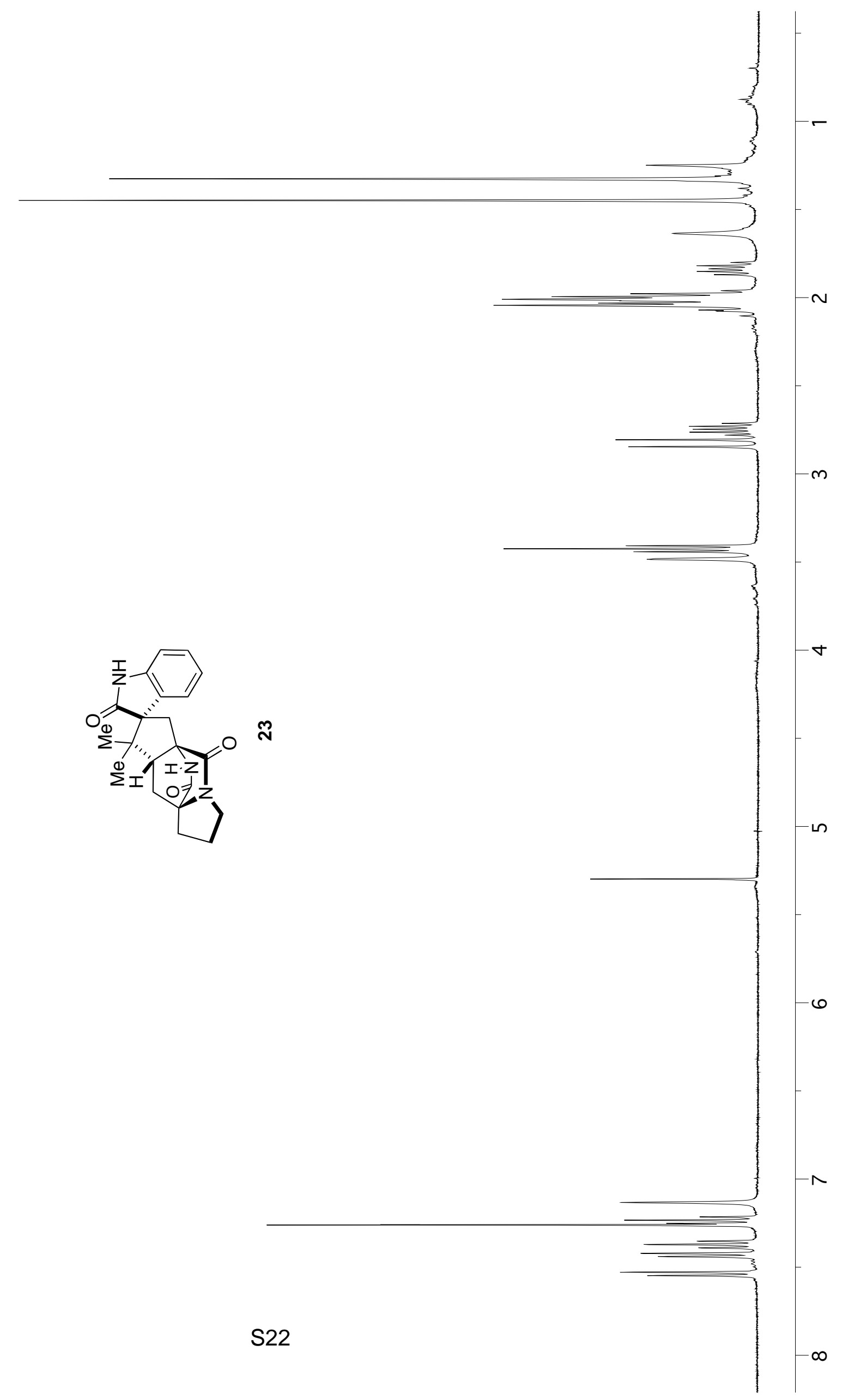


$96 \mathrm{~L}^{\circ} 02$

9\&S" $\downarrow 2$

6St" $\angle 2$

ᄂ69. $2 \varepsilon$

$\varepsilon \varepsilon 0$. $8 \varepsilon$

$8 t 5^{\circ} 0 t$

$0 Z 2 " t t$

$28 t^{\circ} 0 \mathrm{~s}$

$580^{\circ} 29$

$\varepsilon 62^{\circ} \angle 9$

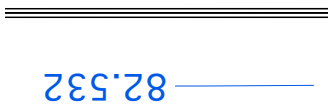

S92.LZL

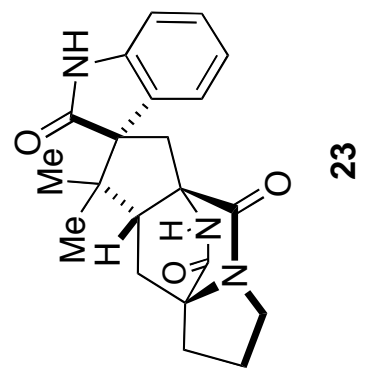

627 ट2L

8EL'9Z L

LSE'OE L

825.0t L

tIL'2SL

SSE'89L

$\angle \forall S^{\circ} Z \angle L$

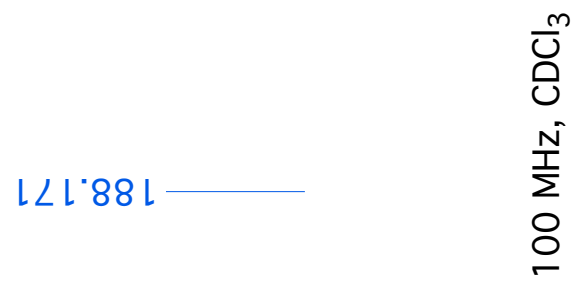



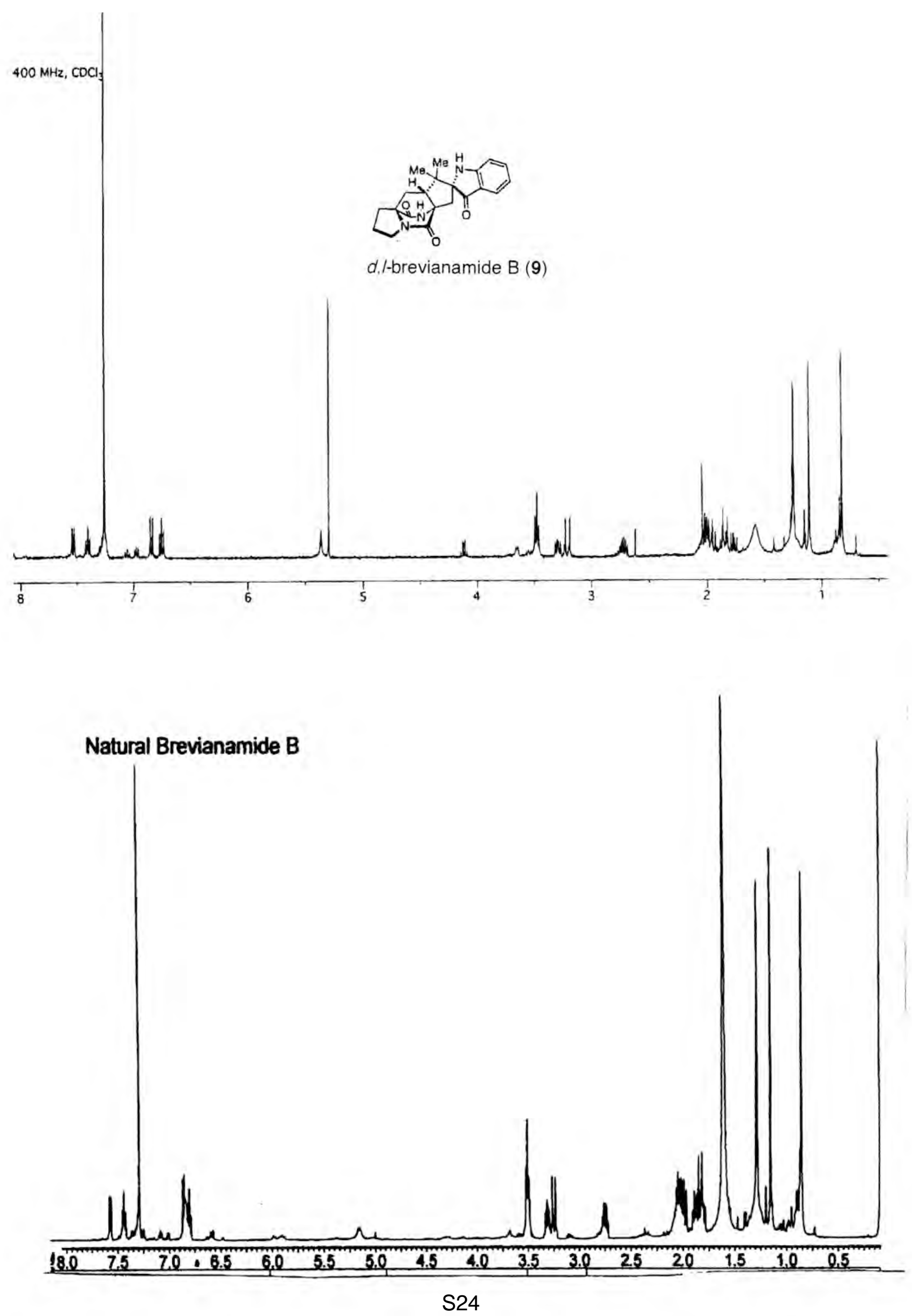